EMBRYARIDDLE
Aeronautical University

SCHOLARLY COMMONS

\section{International Journal of Aviation,} Aeronautics, and Aerospace

\title{
Cruise Missile Integrated Air Defense System Penetration: Modeling the S-400 System
}

\author{
Michael Pelosi \\ East Central University, Ada OK, mpelosi@tamut.edu \\ Amie K. Honeycutt \\ East Central University, Ada OK, amikhon@email.ecok.edu
}

Follow this and additional works at: https://commons.erau.edu/ijaaa

Part of the Navigation, Guidance, Control and Dynamics Commons, and the Systems Engineering and Multidisciplinary Design Optimization Commons

\section{Scholarly Commons Citation}

Pelosi, M., \& Honeycutt, A. K. (2017). Cruise Missile Integrated Air Defense System Penetration: Modeling the S-400 System. International Journal of Aviation, Aeronautics, and Aerospace, 4(3). https://doi.org/ 10.15394/ijaaa.2017.1104

This Article is brought to you for free and open access by the Journals at Scholarly Commons. It has been accepted for inclusion in International Journal of Aviation, Aeronautics, and Aerospace by an authorized administrator of Scholarly Commons. For more information, please contact commons@erau.edu. 
The use of terrain masking techniques to deny detection by surface based sensors, especially radar, has a long and arguably very colorful history. First practiced systematically during the Second World War period, terrain masking relies mostly upon the denial of direct line of sight between the aircraft and the surface based observer, or sensor. The technique was exploited intensively during the Cold War period, with a number of combat aircraft designs optimized for this penetration regime, with specific design optimizations in aerodynamics, propulsion and avionics. Specific types include the F-105 and F-111, and the later Panavia MRCA/Tornado and B-1 (Flight Manual, 1970; 1972).

The advent of modern cruise missiles, such as the AGM-86, BGM-109 and Soviet Kh-55, saw the same penetration regime adopted. As these air vehicles have radar cross section values in applicable radio frequency bands of one to three orders of magnitude smaller than conventional aircraft, low altitude flight, and where feasible terrain masking, significantly improved the survivability of these autonomous weapons (Kopp, 2005-2012; 2009-2012, Riedel et al., 2010).

While denial of direct line of sight is the dominant mechanism in terrain masking, exploiting both the curvature of the earth and frequently elevated terrain features in close proximity to the observer or sensor, it is not the only mechanism of relevance. Radar processing limitations and the physics of low altitude radar propagation can also produce pronounced effects.

Prior to the advent of modern pulse Doppler and Air Moving Target Indicator (AMTI) techniques in radar, targets which were below the line of sight between the radar and the horizon would be obscured by terrain backscatter and termed clutter, making them difficult, if not impossible, to clearly resolve. Target acquisition and tracking performance in such geometries depend strongly upon the specific design of the radar system in question, encompassing antenna, signal processing and tracking algorithm design and integration. Introductory treatments of this problem are presented by Lynch and Kopp, and Stimson (Lynch \& Kopp, 2008, Stimson, 1998).

Even where a radar is capable of rejecting clutter, constructive and destructive interference effects arising between directly incident target backscatter, and target backscatter reflected by terrain, will produce what amount to blind elevation angles, with strong dependencies upon the shaping of the terrain and its electrical properties in proximity to the specular point for backscatter reflected from the terrain surface. In practical terms, this will produce periodic and frequently intensive fading effects, in which the magnitude of the target backscatter fluctuates very strongly, presenting severe difficulties in tracking. The 
seminal works in this area were produced by Blake, with additional treatment by Murrow (Blake, 1969, Murrow, 1990).

While the special cases of a smooth surface and homogenous surface electrical properties, for specular and diffuse surfaces, have been well studied, complex surfaces with locally elevated components and inhomogeneous electrical properties are not analytically tractable, and present good opportunities for numerical modeling.

Numerical modeling techniques present other opportunities in this area of study, especially where Geographical Information Systems (GIS) can be integrated with other simulation algorithms. The availability of GIS terrain elevation datasets of high accuracy significantly simplifies numerical modeling of many problems in this area.

This paper explores the effectiveness of terrain masking techniques in low altitude flight, where a GIS is actively exploited to select an optimal flight-path for concealment of an air vehicle, with a cruise missile chosen as a case study. Algorithms for optimal routing of this kind have previously been explored by Pelosi et al, and Petitt (Pelosi et al, 2012, Pettit, 2004).

The imperative for this research is the advent of specialized air defense systems developed specifically to engage Precision Guided Munitions in their "endgame" terminal trajectories, employing either missiles, guns or both, with radar, thermal imaging or other closed loop tracking systems. Systems in this category are intended to defeat anti-radiation missiles, guided bombs, and cruise missiles, and are often labeled "Counter PGM Systems" (Kopp, 2009-2012; 20092012).

These designs typically employ $\mathrm{X}$-band or $\mathrm{Ku}$-band engagement radars, cued by a S-band or C-band acquisition radar, with a thermal imaging adjunct tracker, the latter sometimes supplemented by a laser rangefinder. Specific design aims typically include the ability to react quickly, and then direct fire at multiple concurrent targets, using cueing and tracking data derived from an acquisition radar on the vehicle itself, or external to the vehicle. Typical battery deployments are four vehicles, with a fifth acquisition radar vehicle and command post to cue and coordinate fire (Kopp, 2009-2012; 2009-2011; 2009-2012).

The survivability of cruise missiles is especially of interest in this context, as they are typically subsonic, fly mostly at low altitudes, are expensive compared to other PGMs, and must penetrate, often, hundreds of miles of contested or 
denied airspace. The survivability of cruise missiles enroute can be maximized by careful planning to remain out of the range of known fixed air defenses, and the most likely or most feasible deployment sites for mobile air defenses. The terminal phase of the flight, where the cruise missile approaches its intended Designated Mean Point of Impact, presents the greatest survivability challenge, as the vehicle will be within direct line-of-sight of any Counter PGM system deployed in very close proximity to the intended target. An additional challenge is that terminal flight trajectory algorithms intended to achieve a desired impact angle may further constrain the terminal trajectory shape, especially for a dive maneuver, where the vehicle must "pop up" to an apogee before it can perform a dive (Riedel et al, 2010).

While a number of strategies intended to maximize survivability in endgame trajectories are feasible, terrain masking intended to minimize exposure time to terminal defenses is especially of interest. This is because reaction times in Counter PGM systems are bounded by several constraints, including the angular sweep velocity of acquisition radars, turret slew times, missile gyroscope spin up times, tracking sensor slew, and acquisition times, and often the reaction times of human operators who must decide whether the acquired target merits engagement. Another factor is weapon fly out time, and minimum engagement range limitations in the defending system. The result of these constraints is that often the window in time where an engagement is feasible will be very short, and any routing strategy which minimizes the duration of this window must in turn maximize the survivability of the air vehicle.

The strategy explored in this study is that of minimizing the probability of air vehicle detection by GIS enhanced terrain masked midcourse routing and GIS enhanced terminal flight-path selection, the latter intended to minimize exposure time during the endgame through terrain masking. To simplify modeling, this study assumes a priori knowledge of the location of all air defense systems enroute and in close proximity to the target. While this cannot be assumed in practice, many modern cruise missile designs incorporate data links for midcourse flight-path and aim-point updates, and carry internally stored GIS datasets to support navigation sensors such as TERCOM. This permits implementations where the location and type of air defenses enroute and near the target could be dynamically updated in flight, with the air vehicle's Operational Flight Program dynamically recomputing optimal routes as updates are received. Where the guidance package does not employ TERCOM, the addition of Flash NVRAM to incorporate additional memory for a stored GIS dataset will not incur unreasonable weight or power penalties. A more expensive strategy would be to equip the air vehicle with an emitter locating system to dynamically identify and 
geolocate any air defense systems which might paint the vehicle enroute, and exploit this information to dynamically alter the route to minimize further exposure, with commensurate weight, power and volumetric penalties.

\section{Literature Review}

\section{Air Defense Systems}

Russia has substantially improved over the air defense systems that were in use, and relatively easily penetrated by the US, during the First Gulf War. As of 2014, there are two main systems in operational use that the US Air Force would have to contend with, the S-300 and S-400. The earlier system is known as the S300, shown in Figure 1, and the newer generation is known as the S-400 which you see depicted in Figure 2. Russia has the S-300 deployed in missile batteries throughout their country, and the $\mathrm{S}-400$ is currently devoted to defending the airspace around the capital city of Moscow, which is regarded as the most effectively defended airspace in the world. Russians are extremely fearful about potentially hostile stealth aircraft penetrations. The concern is obvious given the devotion they have dedicated to technical development over the last several decades. The S-300 system is also in wide use by and available through purchase to China, and the rest of the world, including Iran. China will most likely acquire the S-400 system in the next few years. Any US aircraft or cruise missile trying to enter Russian or Chinese airspace will have to contend with detection and missile launch from these extremely capable systems.

Both the S-300 and S-400 equipment systems are organized into air defense batteries, each of which forms a part of a centrally controlled integrated air defense system, or IADS, of a region. Each battery consists of about 6-8 vehicles. There is one early warning or acquisition radar vehicle, which may also be the headquarters vehicle for the battery, there is an engagement radar vehicle, and about four to six transporter / erector / launcher (TEL) vehicles, which each transports 4 surface-to-air missiles in tubes on the back of the vehicle. 


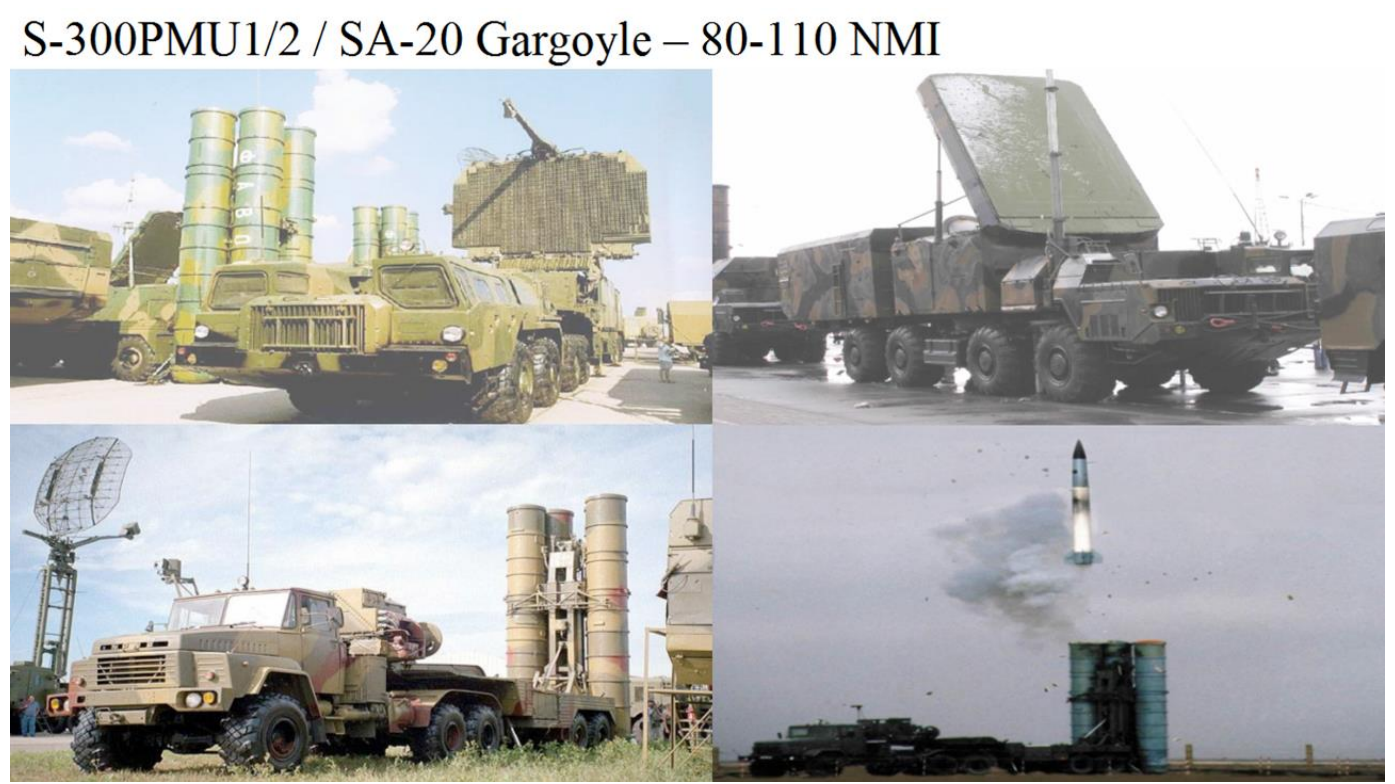

Figure 1. S-300 System.

The acquisition radar constantly searches the sky out to several hundred miles around the battery location since the engagement radar can only observe a very narrow section of the sky by design. Once the acquisition radar locates a potential target and confirms it, it queues up the engagement radar which will get a precision location and heading to the target, at which time missiles can be launched and are guided most of the way to the target, at which time the missile's internal seeker algorithms and sensors take over and perform terminal missile guidance to impact the target. The entire sequence between initial detection, missile launch, guidance, and impact can take 30 to 60 seconds. This is why when deployed to the field, IADS missile batteries are on a virtual hairpin triggers around-the-clock, as every second can make the difference regarding missing an incoming aircraft which would then be able to launch and deliver its weapons successfully. For the S-300 system, the acquisition radar is the 64N6 Big Bird shown in the upper left in Figure 1, in the upper right is the 30N6 Tombstone engagement radar, the truck in the lower left is the four missile round Transporter Erector Launcher (TEL) vehicle, shown with the missile tubes prepared for launch, and the 48N6 missile used is shown being launched in the lower right of Figure 1. It is strikingly apparent from the size of the missile that the explosive payload $(145 \mathrm{~kg})$ is easily more than enough to bring down just about any aircraft: military, civilian, or commercial. 


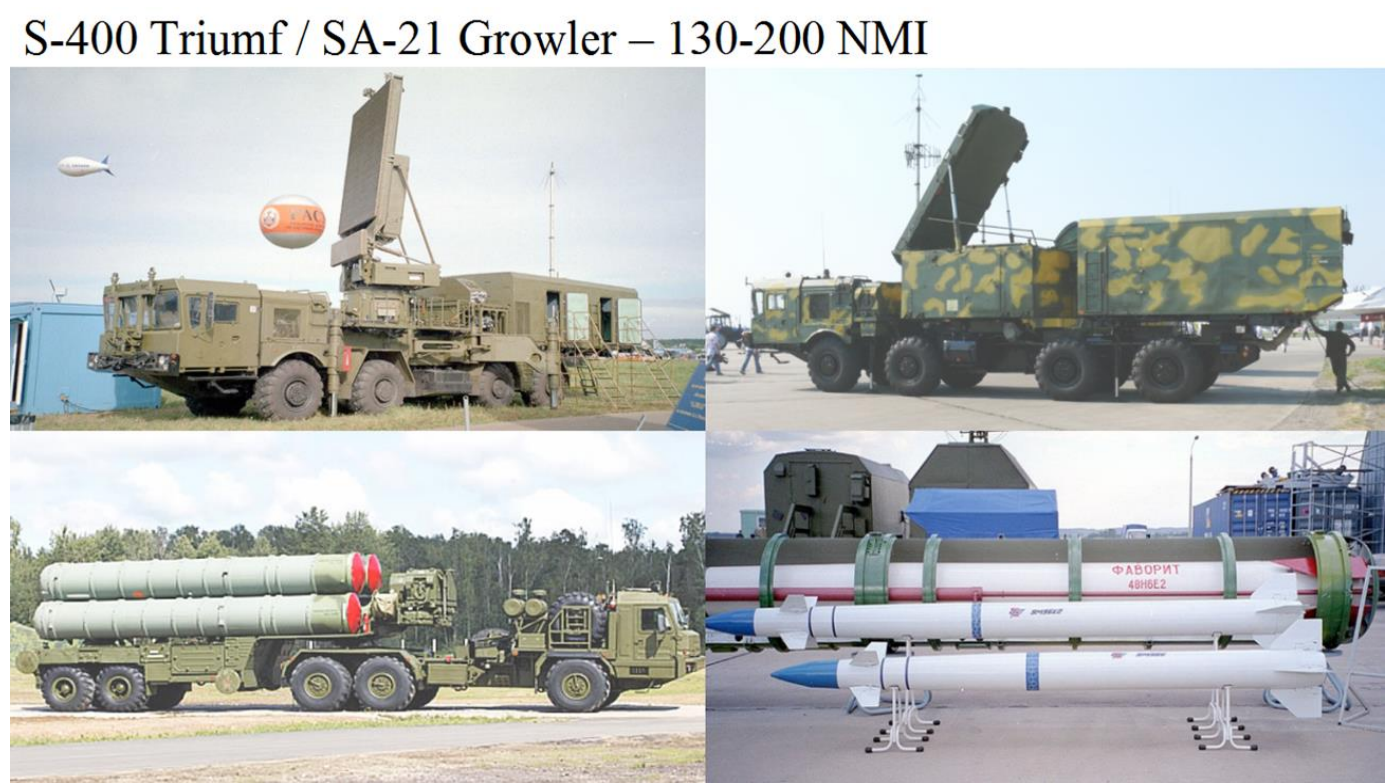

Figure 2. S-400 System.

Shown above in Figure 2 is the even more modern and capable S-400 Triumf system. This system consists of the 96L6 Cheese Board acquisition radar shown in the upper left, the 92N2 Grave Stone engagement radar shown in the upper right, the 4 round transporter erector launcher (TEL) vehicle shown in the lower left, and the $48 \mathrm{~N} 6$ surface-to-air missile shown in the lower right. The range of the $48 \mathrm{~N} 6$ missile is about 250 miles, so $4 \mathrm{~S}-400$ batteries could cover approximately 1,000 miles of air frontage. Longer-range missiles, such as the 40M6, are also available. Typically, when launching a missile at a target, a single launch round will suffice, except for protocol adaptations such as in the case of a high-value B-2 bomber, in which situation two missiles may be launched to further increase the probability of successful hits. Since the 48N6 missile travels at Mach 12.0, it will cover 250 miles in about 98 seconds.

Russia has had focused development to improve these radars and missiles so that it is virtually impossible for older generations of aircraft, such as the F-14, F-15, F-16, and F/A-18 to successfully penetrate air spaces defended by the S-300 and S-400 systems. It is widely regarded in defense circles, therefore, that these aircraft should be considered obsolete - they are not survivable in a modern venue. Only the F-22, B-2, to a lesser extent the F-35, and some of the cruise missiles such as the Tomahawk are the only strong choices capable of penetrating the airspace defended by these two systems. Any technological edge the US can procure to reduce the probability of detection (and thereby possible destruction) 
of their most capable aircraft by these air defense systems is sorely needed given the lack of new aircraft and missile development by the US.

The best outcome for a US penetrating aircraft or missile would be to simply avoid detection by any of these system's radar while flying its mission. Leveraging stealth is now more important than ever in the second decade of the 21 st century, but as these air defense systems become more capable, that prospect is becoming much more elusive.

Russia has also developed an impressive portfolio of additional radar systems specifically designed to detect low-flying and stealth aircraft and cruise missiles which you can see some examples of listed below in Figure 3.

\begin{tabular}{|c|c|c|c|c|c|c|}
\hline Radar Type & NATO Designation & RF Band & SAM System & NATO Designation & Role/Function & Status \\
\hline P-18 & Spoon Rest D & VHF & S- 75, S-125 & SA-2, SA-3 & Acquisition & Legacy/Upgrade \\
\hline $1 L 13$ Nebo SV & - & VHF & S-300PMU/S-400 & SA-20, SA-21 & Acquisition & Production \\
\hline 1 L119 Nebo SVU & 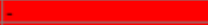 & VHF & S-300PMU/S-400 & SA-20, SA-21 & Acquisition & LRIP \\
\hline 5N84AE Oborona-14 & Tall King & VHF & S- 75, S- -125, S- 200 & SA-2, SA-3, SA-5 & Acquisition & Legacy/Upgrade \\
\hline 5Zh66 Nebo UE & Tall Rack & VHF & S -400 & $\mathrm{SA}-21$ & Acquisition & Production \\
\hline P-15/15M/19 & Flat Face / Squat Eye & UHF & S- 75, S-125 & SA-2, SA-3 & Acquisition & Legacy/Upgrade \\
\hline 51U6 Kasta 2E1/2E2 & - & UHF & S- 75, S- 125 & $\mathrm{SA}-2, \mathrm{SA}-3$ & Acquisition & Production \\
\hline 67N6E Gamma DE & - & L & S-300PMU/S-400 & SA-20, SA-21 & Acquisition & Production \\
\hline 59N6E Protivnik-GE & - & L & GCI & - & Acquisition & \\
\hline P-35M/37/1L117 & Bar Lock & $\mathrm{s}$ & GCI & - & Acquisition & Legacy/Upgrade \\
\hline 96L6E & Cheese Board & $\mathrm{s}$ & S-300PMU/S-400 & SA-20, SA-21 & Acquisition & Production \\
\hline $5 \mathrm{~N} 66 \mathrm{M} / 7 \mathrm{~N} 6$ & Clam Shell & & - & SA-10, SA-20 & Acquisition & Production \\
\hline 36D6/ST-68M & Tin Shield & $\mathrm{s}$ & S-300PT/PS/PMU & SA-10, SA-20 & Acquisition & Legacy/Upgrade \\
\hline 64N6E/E2 & Big Bird & $\mathrm{s}$ & S-300PMU1/PMU2 & SA-20 & Acquisition & Production \\
\hline 92N6E & Big Bird & Big Bird & $S-400$ & SA-21 & Acquisition & Development \\
\hline P-35M/37 & Bar Lock & $\mathrm{s}$ & S- 75, S- 125 & SA-2, SA-3 & Acquisition & Legacy/Upgrade \\
\hline TRS-2100 Tiger S & - & $\mathrm{s}$ & - & & Acquisition & Production \\
\hline 9S15M Obzor-3 & Bill Board & $\mathrm{s}$ & S-300V/VM & SA-12/23 & Acquisition & Production \\
\hline 9S18 Kupol & Tube Arm & $\mathrm{s}$ & $9 \mathrm{~K} 37 \mathrm{M}$ Buk & SA-11 & Acquisition & Legacy/Upgrade \\
\hline 9S18M1 Kupol-M & Snow Drift & $\mathrm{s}$ & 9K37M1 Buk-M1 & SA-17 & Acquisition & Production \\
\hline 1RL-144 Tunguska $M$ & Hot Shot & $\mathrm{s}$ & 9M311 & SA-19 & Acquisition & Production \\
\hline 1RL-144M Pantsir S1 & Hot Shot & $\mathrm{s}$ & $9 \mathrm{M} 335 / 57 \mathrm{E} 6$ & SA-22 & Acquisition & Production \\
\hline SNR-75 & Fan Song & $\mathrm{S} / \mathrm{X}$ & S-75 & SA-2 & Engagement & Legacy/Upgrade \\
\hline $1 \mathrm{~S} 91$ & Straight Flush & $\mathrm{S} / \mathrm{X}$ & ZRK Kub & SA-6 & Acquisition & Legacy/Upgrade \\
\hline 4R33 Baza & Land Roll & $\mathrm{s} / \mathrm{X}$ & 9A33BM3 ZRK Romb & SA-S & Acquisition & Legacy/Upgrade \\
\hline 1591 & Straight Flush & $\mathrm{x}$ & ZRK Kub & SA-6 & Engagement & Legacy/Upgrade \\
\hline $5 \mathrm{~N} 62$ & Square Pair & $\mathrm{x}$ & S-200 & SA-5 & Engagement & Legacy \\
\hline 9S19M2 Imbir & High Screen & $\mathrm{x}$ & S-300V/VM & $\mathrm{SA}-12 / 23$ & Acquisition/ABM & Production \\
\hline 30N6E & Flap Lid & $\mathrm{x}$ & S-300PT/PS/PM & SA-10 & Engagement & Legacy/Upgrade \\
\hline 30N6E1 & Tomb Stone & $\mathrm{x}$ & S-300PMU1 & SA-20 & Engagement & Legacy/Upgrade \\
\hline 30N6E2 & Tomb Stone & $\mathrm{x}$ & S-300PMU2 & SA-20 & Engagement & Production \\
\hline 92N2E & Grave Stone & $\mathrm{x}$ & S -400 & $\mathrm{SA}-21$ & Engagement & Production \\
\hline $9 \mathrm{~S} 32$ & Grill Pan & $\mathrm{x}$ & S-300V/VM & SA-12/23 & Engagement & Production \\
\hline 9S35M1 & Fire Dome & $\mathrm{x}$ & Buk-M1 9K37M1 & $\mathrm{SA}-11 / 17$ & Engagement & Production \\
\hline SNR-125 & Low Blow & $\mathrm{x}$ & S-125 & SA-3 & Engagement & Legacy/Upgrade \\
\hline RPK-2 Tobol & Gun Dish & $\mathrm{x}$ & ZSU-23-4P & ZSU-23/4P & Engagement & Legacy/Upgrade \\
\hline Tor/Tor M1/M2 & Scrum Half & $\mathrm{x}$ & 9A331M & $\mathrm{SA}-15 \mathrm{~A} / \mathrm{B} / \mathrm{C}$ & Acquisition & Production \\
\hline 4R33 Baza & Land Roll & $\mathrm{x} / \mathrm{Ku}$ & 9A33BM3 ZRK Romb & SA-8 & Engagement & Legacy/Upgrade \\
\hline Tor $/$ Tor $\mathrm{M} 1 / \mathrm{M} 2$ & Scrum Half & $\mathrm{K}$ & 9A331M & $\mathrm{SA}-15 \mathrm{~A} / \mathrm{B} / \mathrm{C}$ & Engagement & Production \\
\hline 1RL-144 Tunguska M & Hot Shot & $\mathrm{Ka}$ & $9 \mathrm{M} 311$ & SA-19 & Engagement & Production \\
\hline 1RL-144M Pantsir S1 & Hot Shot & $\mathrm{Ka}$ & 9M335 / 57E6 & SA-22 & Engagement & Production \\
\hline
\end{tabular}

Figure 3. Operational Russian Radar Systems (source: www.ausairpower.net).

The list above makes evident the wide coverage spectrum coverage resulting from the various radars through the frequency bands of VHF, UHF, Lband, S-band, X-band, Ku-band, K-band, and the Ka-band. Russia has plentiful designs in all frequency ranges, and large quantities of deployed systems. These together can be considered quite a daunting array of long- and medium-range 
radar systems that an Air Force may be called upon to attempt to defeat with a very limited number of available and capable aircraft.

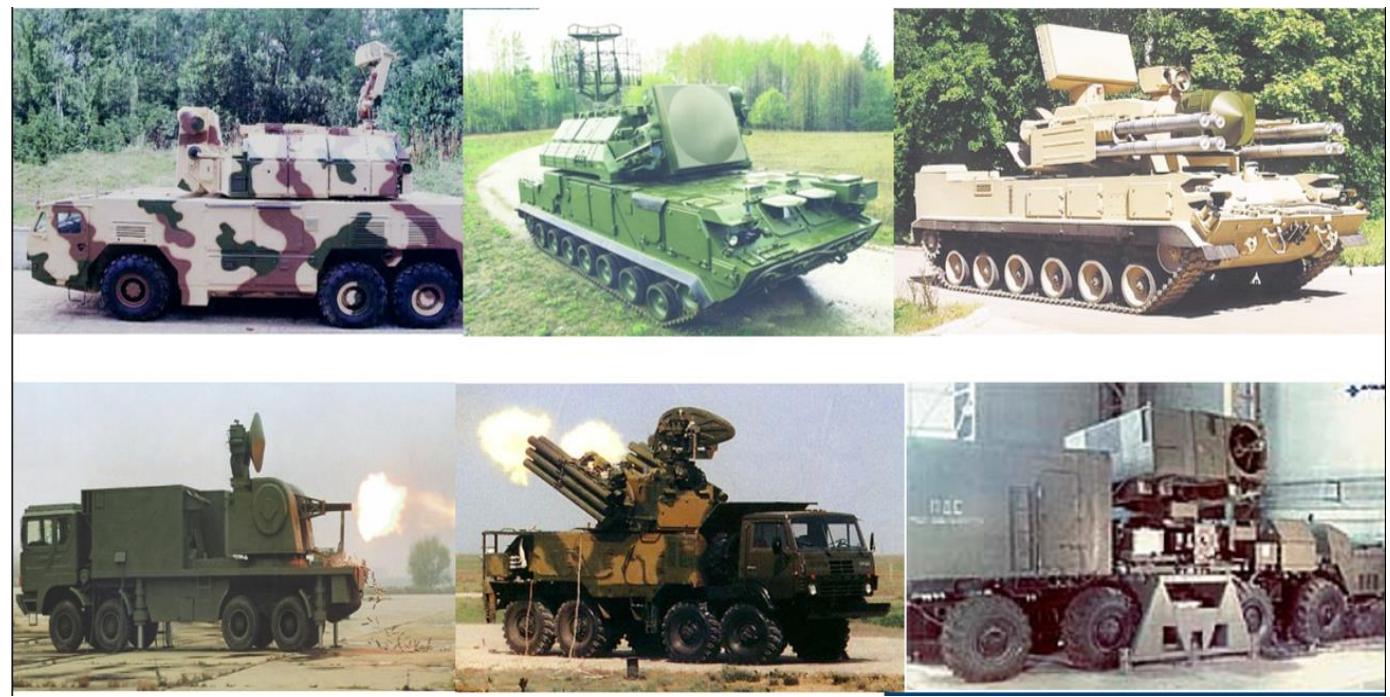

Figure 4. Russian Point Defense Systems.

In addition to the long-range systems discussed, there are also systems designed for what is known as short range "point defense". If any missile or aircraft gets through the longer-range S-300 or S-400 type systems, these point defense systems are optimized for extremely quick responses and shoots within a few seconds. They are also much more mobile than the longer-range systems, and move around frequently by design and doctrine.

Some of these point defenses depicted in Figure 4 include the Tor M2, Tor M1, Tungusca M, the LD-2000 in the lower left, Pantsir S2, and in the lower right the Almaz-Antey laser directed energy weapon development project. This is quite an array of additional air defense assets that the US may be called upon at some time to deal with, and it includes various long-range search and early warning radars, missile battery system such as the S-300 and S-400, the wide assortment of point defense weapons, and laser directed energy weapon systems under development.

At the same time as being defended by very capable IADS systems, many of the targets themselves will be protected in super-hardened underground shelters, such as in the case of the more than 45 PLAAF underground mountain bases, which in total comprise enough sheltering area to hold within them more than the entire 1,500 strong PLAAF aircraft fleet simultaneously. 
The most capable aircraft the US would have for the foreseeable future for likely deep-penetration missions include 16 B-2 bombers and 187 F-22 fighters. Since the available number of these aircraft will be not sufficient for a protracted campaign. One solution would be maximizing the effectiveness of available cruise missiles, such as the Tomahawk cruise missile, a 1980s Cold War design. The numbers of these would likely be severely limited also, but a compensating prospect is that production could be ramped up on a much shorter than for aircraft production. Additionally, each missile costs a fraction of combat aircraft cost, usually on the order of 1/100th, or quite a bit less, than the cost of a single combat aircraft. The Tomahawk missile, according to the US Navy, costs $\$ 569,000$ (United States Navy). In comparison, the Government Accounting Office (GAO) reports the unit cost of F-35 to be $\$ 142.6$ million (Sullivan, 2014). That is less than $1 / 200$ th of the aircraft cost.

As a result, our research presented in this paper focuses on potentially improving the penetration and survivability of the Tomahawk or other cruise missiles into the airspace defended by modern IADS systems.

In summary this is an extremely high-stakes game of air defense, and attack, as well as stealth, that nations in the global community have been working on to outclass each other in for a long time spending in total hundreds of billions of dollars. Unfortunately, the balance has tipped recently more towards the favor of non-US/NATO bloc aligned countries.

\section{Mission Route Planning}

This section focuses closely on the technical aspects of how to accomplish the stated research goal, given the likely parameters that we have previously discussed regarding the penetration of a modern IADS. As previously mentioned, US forces are, with little doubt, constrained extensively by the small number of available and capable aircraft in future decades for these types of missions. Not only is this for possible actual missions, but also useful in attempting to maintain the deterrence effect of US military power. US forces should at least have the potential to run these missions successfully with little doubt in order to prevent unnecessary foreign aggression.

Computer science and artificial intelligence techniques can maximize the effectiveness of some of the available equipment, even if that equipment is less than ideal as previously mentioned. The goal here is to increase the efficiency of existing and available equipment with only small added financial costs while trying to maintain a short development time. Developing new aircraft typically 
takes 1 to 2 decades, and recently, even longer. New development costs are not something that will be able to be shouldered within the federal budget again anytime soon, with limited exceptions. In other words, hardware that is currently in the arsenal may very well be all that is available on short notice, and leveraging it effectively should be a high priority for the US military.

One alternative to using long-range penetrating aircraft is precision guided munitions, such as the Tomahawk cruise missile. One attractive aspect of using a design such as the Tomahawk is that it is able to be mass-produced and production could be ramped up theoretically in several months. Also, the price tag is significantly lower than any of the aircraft at only $\$ 1.5$ million, so it is the equivalent of 1/100th of an F-22 or 1/500th of a B-2 bomber. The Tomahawk entered service in 1983. It is not stealthy by design, nor is it supersonic, like the newer generation Russian and Chinese cruise missiles. As a result, it has some significant limitations, such as that the slow speed which makes it particularly vulnerable to point defenses, many of which we mentioned earlier. These can catch a slow moving cruise missile, hugging the ground at treetop heights, in its final few seconds before it will impact its target. The point defenses will be grouped right around the general area of the target specifically scanning the horizon for incoming cruise missiles.

The research problem posed more specifically therefore is how do we enhance the survivability and effectiveness of the Tomahawk cruise missile by using computer science techniques? Figure 5 shows more details on the Tomahawk BGM-109, also known as the TLAM.

The TLAM uses a GPS system for navigation and guidance, it is 18 feet long, has a wingspan of 8 feet, and weighs 2,700 pounds. It has a range of 700 to 1000 miles, and flies at a speed of $550 \mathrm{mph}$. It has a 1,000-pound conventional warhead. These missiles can be launched from submarines, cruisers, destroyers, and even the ground. The missile is designed to fly above the terrain at an altitude as low as 50 feet using stored elevation maps and the onboard radar and GPS. An onboard camera compares the actual target to a stored image as it zeroes in on the final approach. One way to avoid radar detection is to fly at ground level, such as the TLAM does, since a radar will be blinded to any objects behind blocking terrain obstacles or topography. So the general approach is to find a path that minimizes the risk to the TLAM through the air defense system, utilizing and taking advantage of some computer science and artificial intelligence techniques known as traversal algorithms. 


\section{Tomahawk BGM-109 Cruise Missile (TLAM)}

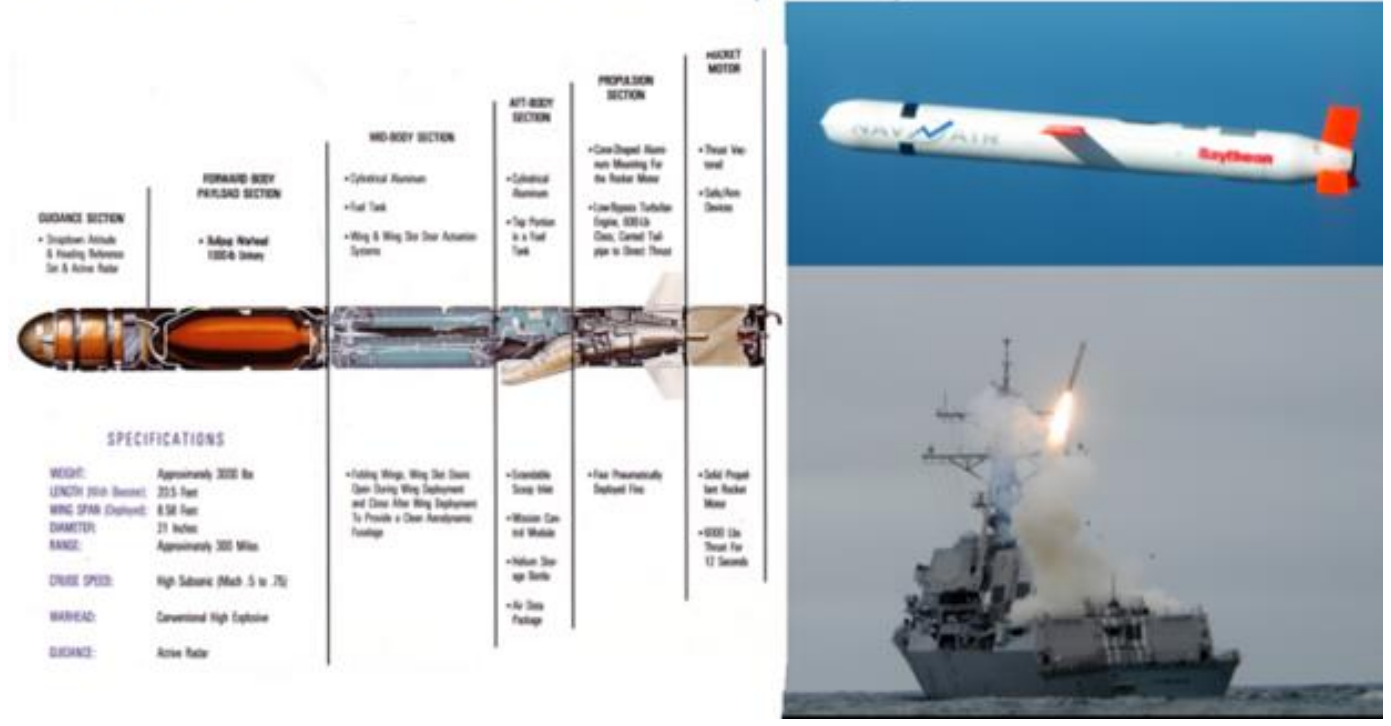

Figure 5. Tomahawk BGM-109 Cruise Missile.

In computer science, graph traversal is the problem of visiting all the nodes in a graph in a particular manner updating and/or checking their values along the way. Tree traversal is a special case of graph traversal. In our case our terrain map around the target is our graph, and we want to traverse this graph in a manner so as to reduce radar detectability of the TLAM from different radar sites that are located on the map.

\section{S-400 and Pantsir Specific Performance and Constraint Modeling}

Practical mission planning experience in given terrain situations dictates there is simply no way to avoid radar detection, especially in areas up close and near to well defended targets. Opportunities for terrain masking may be nonexistent. However, the pathfinding algorithm can exploit time delays and equipment limitations of the various systems if these costs are modeled and specifically defined for the algorithm computation. This prospect could be seen as analogous to the Cowboy Western movie situation where the gunfighters hide behind a rock and dash to another location before the opponent can muster their aim well enough to fire with any accuracy. If an enroute TLAM cruise missile can find another obstacle to hide behind before the SAM shot can be gotten off successfully, even though in this case the missile did not avoid detection, it will still be survivable. 
Shown below in Figure 6 are the distilled out relevant timing and equipment performance constraints for the $S-400$ that the system must conform to in order to engage targets successfully. For example, before a missile can be launched, a sequence of complex events has to occur, each taking at least a minimum amount of time. The early warning radar has to spot the missile and hand it off to the separate engagement radar, each of these requires additionally at least two sweeps of radar to adequately track a TLAM or other cruise missile. Handoff from one radar to the other takes a few seconds, as well as staff evaluation and decision as to whether the target is legitimate and worthy to be fired upon. If the IADS sector had been previously quiet, the missile gyros may not have been previously started, and it takes approximately 10 seconds for the SAM missile gyros to spin up before a missile can be launched.

Altogether for the S-400 system, our analysis shows it would take between 54 and 151 seconds for the battery to successfully impact a target with a missile. Detection and tracking could be lost at any time during the sequence, at which time the TLAM missile would have to be reacquired and the sequence started from scratch. By having the movement pathfinding algorithm account for these time delays, and assign much higher cost multipliers for movements later in any potential engagement sequence, movements with high risks of shoot-down can be avoided relative to the trade-offs available on different routes.

The sequences, depicted in Figure 6, include: possible early warning detection, which is best case for the missile battery to be ready with missile gyros spun up, a missile battery tracking activity and launch decision, and the missile launch and fly out activities, which also require time and continuous consecutive tracking for most of the missile flight time. During the last few seconds of SAM missile flight, the missile's internal guidance and navigation system takes over to impact the target with no additional tracking guidance provided by the battery. However, even at this time, a clear line-of-sight must be maintained between the SAM and the TLAM until impact. These sequences are encoded into the calculations of our mission planning model algorithms. 


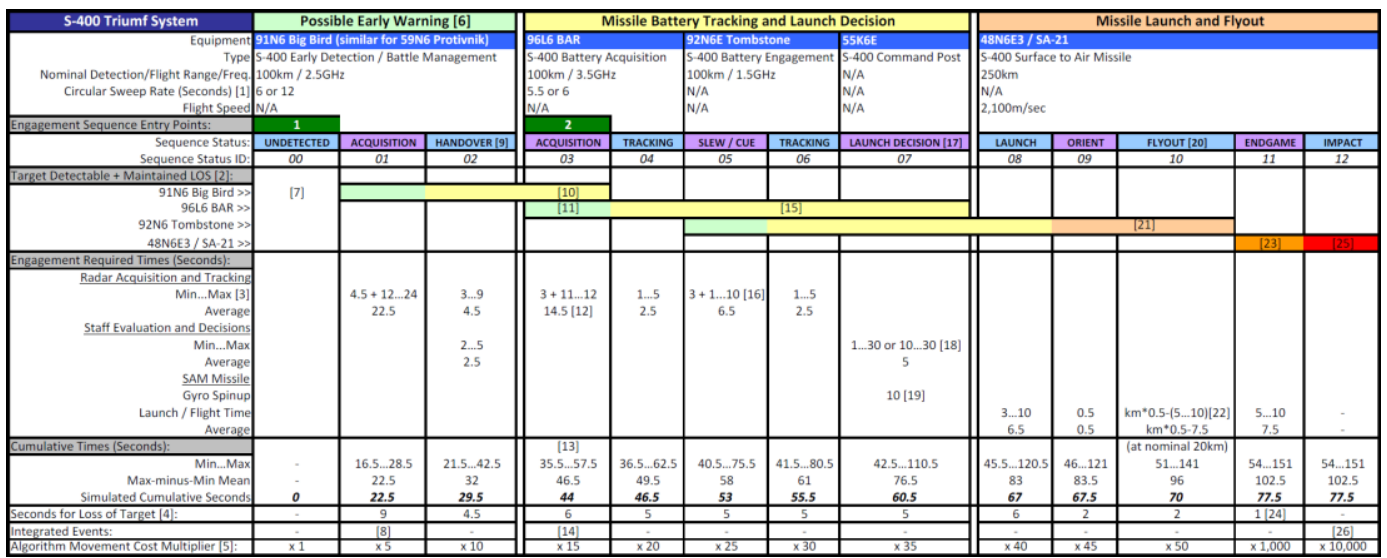

Figure 6. S-400 System Engagement and Launch Process (notes in [ ] brackets below).

S-400 acquisition, tracking, engagement, and launch sequence checkpoints and description notes from Figure 6:

1. A minimum of two radar sweeps is required to detect and acquire a target.

2. "Detectable" is defined as the radar cross section aspect shown of the target having a probability of detection greater than $90 \%$ for given radar equipment performance specifications. "LOS" is defined as a clear straight-line path ("line-of-sight") existing between the radar and the target containing no terrain or other obstructions.

3. For radar sweeps, preliminary value is 180-degree average sweep/slew distance to be covered before first target return (90 degrees in case of $91 \mathrm{~N} 6$ as it is dual-faced).

4. This is the loss of consecutive target track time that makes it necessary for the sequence be started from scratch at Entry Point 1 or 2 in order to reacquire a target.

5. The danger-escalation cell movement cost "penalty" multiplier for the A* Algorithm that is used for potential movement evaluation during each status indicated.

6. Early detection may take place if the TLAM is observable within range and LOS of any system early detection radar.

7. The TLAM will remain undetected by flying behind terrain masking and/or remaining out of detectable radar range.

8. In this case: A. Brigade command post notified. + B. All IADS assets put on sector alert. + C. All system missile gyros are spun up.

9. This phase involves delay for human interpretation and decision.

10. 91N6 Big Bird and 96L6 BAR detection should be concurrent at this point for most efficient handover. 
11. 96L6 BAR can autonomously acquire targets with 2 radar sweeps of a detectable object.

12. Half this value (7.25 seconds) is used if Early Warning exists.

13. With Sequence Entry Point 2, cumulative time value at Handover (29.5 Seconds) can be subtracted.

14. For autonomous battery acquisition, missile gyro spin up will occur at this time.

15. 96L6 BAR and 92N6E Tombstone detection should be concurrent until launch decision is complete.

16. 3 second average initial slew is removed if Early Warning exists.

17. Possible existence of an S-400 "full automatic" mode may preclude any battery decision delays if it is activated.

18. 10-30 seconds if no Early Warning and/or missile gyro spin up is necessary.

19. Necessary if no Early Warning--may or may not be on critical time path for missile launch

20. The 48N6E3 missile guidance control algorithm uses a modified p-Nav approach where missile aims for projected target location at time of impact.

21. Only 92N6E Tombstone tracking is necessary during missile fly out, however a 2 second detection loss will result in errant missiles that do not impact the target.

22. This includes 5\%-time penalty for missile control maneuvering delays and non-direct flight. 7.5 seconds is subtracted for average Endgame phase length.

23. No radar tracking is necessary during final 7.5 seconds of missile endgame for selectable missile types.

24. Loss of target to radar track during missile endgame is not relevant-missile relies at this point on internal TVM/SAGG guidance. LOS to missile location is evaluated instead in Endgame.

25. Salvos of 2 missiles are normally fired, making the probability of missile misses insignificant for a slow-moving TLAM type target.

26. TLAM destruction with probability of $100 \%$ if prior conditions are achieved.

As a specific example of one of the many constraints we have discussed for tracking and firing of SAM missiles, Figure 7 shows the multiple sweep radar tracking problem. The radar will need multiple sweeps to get a reliable detection, and many sweeps to effect track and cue for engagement, for the fire control radar to effect a missile shot. The beam sweep time is determined by the number of revolutions per minute of the radar equipment which is fixed based on the motor rotational speed turning the radar antenna and cannot be changed. 


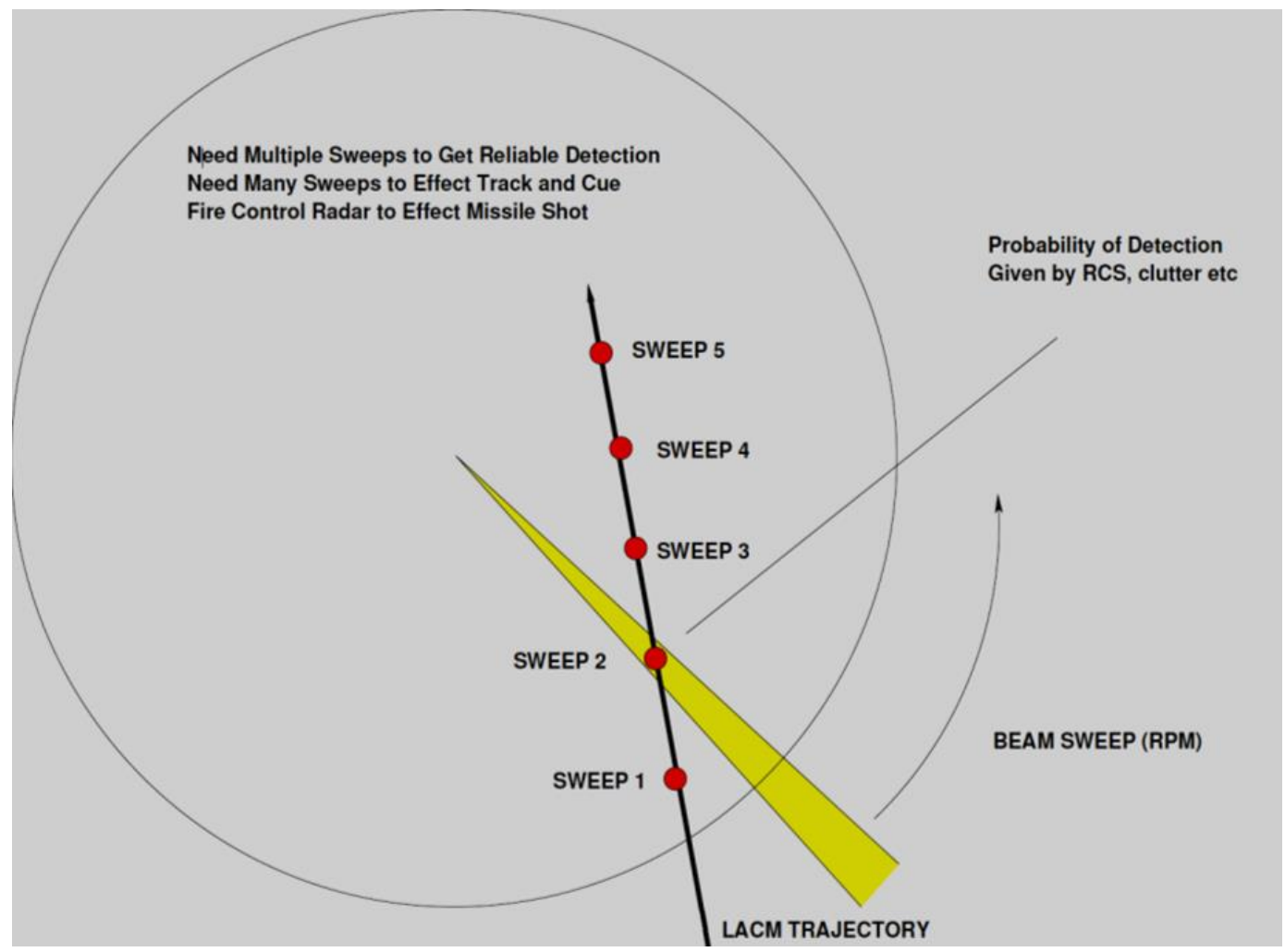

Figure 7. The Multiple Sweep Radar Tracking Problem.

Similar to the breakdown for the S-400 system, the table in Figure 8 below shows the constraints and time delays for the Pantsir point defense system. It is calculated that this system would take between 47 and 98 seconds from the time of first detection to missile impact, with a most likely case of about 64 seconds.

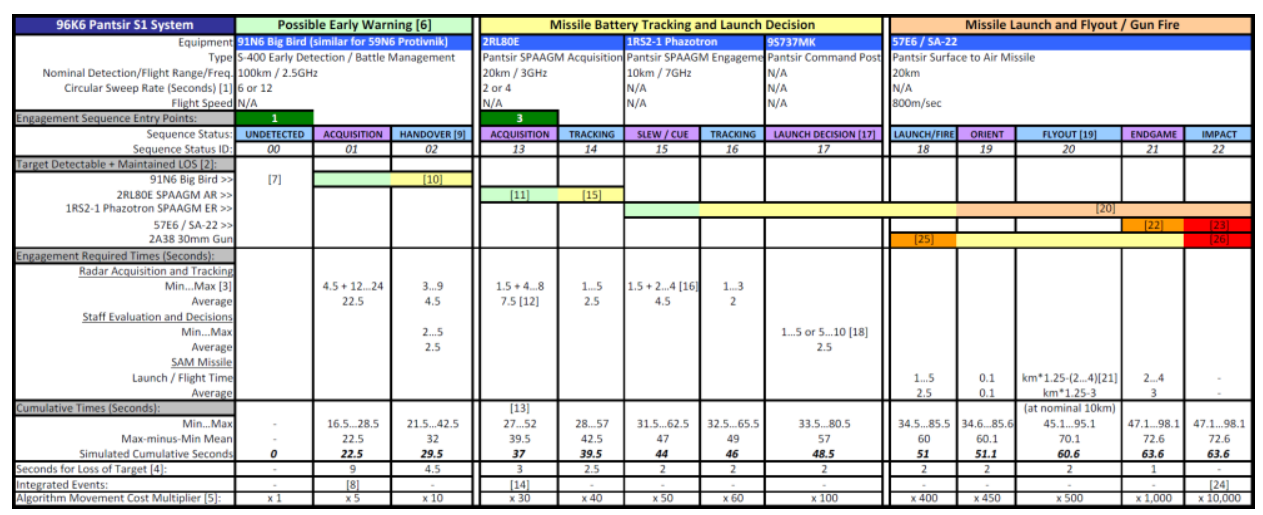

Figure 8. Pantsir System Engagement and Launch Process (notes in [ ] brackets below). 
Pantsir acquisition, tracking, engagement, and launch sequence checkpoints and description notes from Figure 8:

1. A minimum of two radar sweeps is required to detect and acquire a target.

2. "Detectable" is defined as the radar cross section aspect shown of the target having a probability of detection greater than $90 \%$ for given radar equipment performance specifications. "LOS" is defined as a clear straight-line path ("line-of-sight") existing between the radar and the target containing no terrain or other obstructions.

3. For radar sweeps, preliminary value is 180-degree average sweep/slew distance to be covered before first target return (90 degrees in the case of $91 \mathrm{~N} 6$ as it is dual-faced).

4. This is the loss of consecutive target track time that makes it necessary for the sequence be started from scratch at Entry Point 1 or 3 in order to reacquire a target.

5. A danger-escalation cell movement cost "penalty" multiplier for the $A^{*}$ Algorithm. Used for potential movement evaluation during each status indicated.

6. Early detection may take place if the TLAM is observable within range and LOS of any system early detection radar.

7. TLAM will remain undetected by flying behind terrain masking and/or remaining out of detectable radar range.

8. At this point: A. Brigade command post notified. + B. All IADS assets put on sector alert.

9. This phase involves delay for human interpretation and decision.

10. 91 N6 Big Bird and 2RL80E detection does not need to be concurrent after this point for handover to occur.

11. 2RL80E can autonomously acquire targets with 2 radar sweeps of a detectable object.

12. Half this value ( 3.75 seconds) is used if Early Warning exists.

13. With Sequence Entry Point 3, cumulative time value at Handover (29.5 Seconds) can be subtracted.

14. For autonomous battery acquisition, missiles are readied at this time.

15. 2RL80E and 1RS2-1 detection does not need to be concurrent after this point.

16. 1.5 second average initial slew is removed if Early Warning exists.

17. Possible existence of a Pantsir S1 "full automatic" mode may preclude any battery decision delays if it is activated.

18. 1-5 seconds if no Early Warning is necessary. 
19. The 57E6 missile guidance will aim for projected target location at time of impact.

20. Only 1RS2-1 Phazotron tracking is necessary during missile fly out, however a 2 second tracking loss will result in errant missiles that do not impact the target.

21. Includes a 0\%-time penalty for missile control maneuvering delays and non-direct flight. 3 seconds is subtracted for average Endgame phase length.

22. Radar tracking continues to be necessary during final 3 seconds of missile endgame.

23. Salvos of 2 missiles are normally fired, making the probability of missile misses insignificant for a slow-moving TLAM type target.

24. TLAM destruction with probability of $100 \%$ if previous conditions are achieved.

25. At maximum range $(4 \mathrm{~km}), 30 \mathrm{~mm}$ gun fire will take approximately 4 seconds to close the distance to the target at $980 \mathrm{~m} / \mathrm{s}$. Target must remain in sight during this entire period.

26. TLAM destruction with probability of $100 \%$.

\section{Results}

Figure 9, below, shows two sample completed mission routes. The start location is in the lower left and the goal location is in the center of the map. Fourpoint defense systems are shown at the centers of the red range circles. There also two longer range S-400 radars in the upper right and lower right of the terrain area. These paths give a good idea of the sensitivity of the path to very small changes. The path on the left is $29 \mathrm{~km}$ long, and has 12 seconds during which time the incoming TLAM missile would be detected. The path on the right is also $29 \mathrm{~km}$ in length but has reduced the time of detectability down to one second. Each of these paths are flying within a few hundred meters of the other, but that small difference is enough to shield the missile using the terrain features from virtually assured destruction.

The locations of the SAM batteries and radars may not be known before a mission. The mission route planners would in this case be compiling information using an accumulated dossier of satellite reconnaissance, aircraft reconnaissance, human intelligence, and signal intelligence by picking up the radar emissions from the various sites and triangulating them. Unfortunately, defending air defense vehicles will be moving around often as part of their doctrine. As a result, the intelligence of radar deployments will be limited at best, mission flight paths may be necessarily re-planned in-flight to account for new information. The entire 
intelligence picture will not be known on most occasions. Routes can be recalculated in progress using newly derived information.

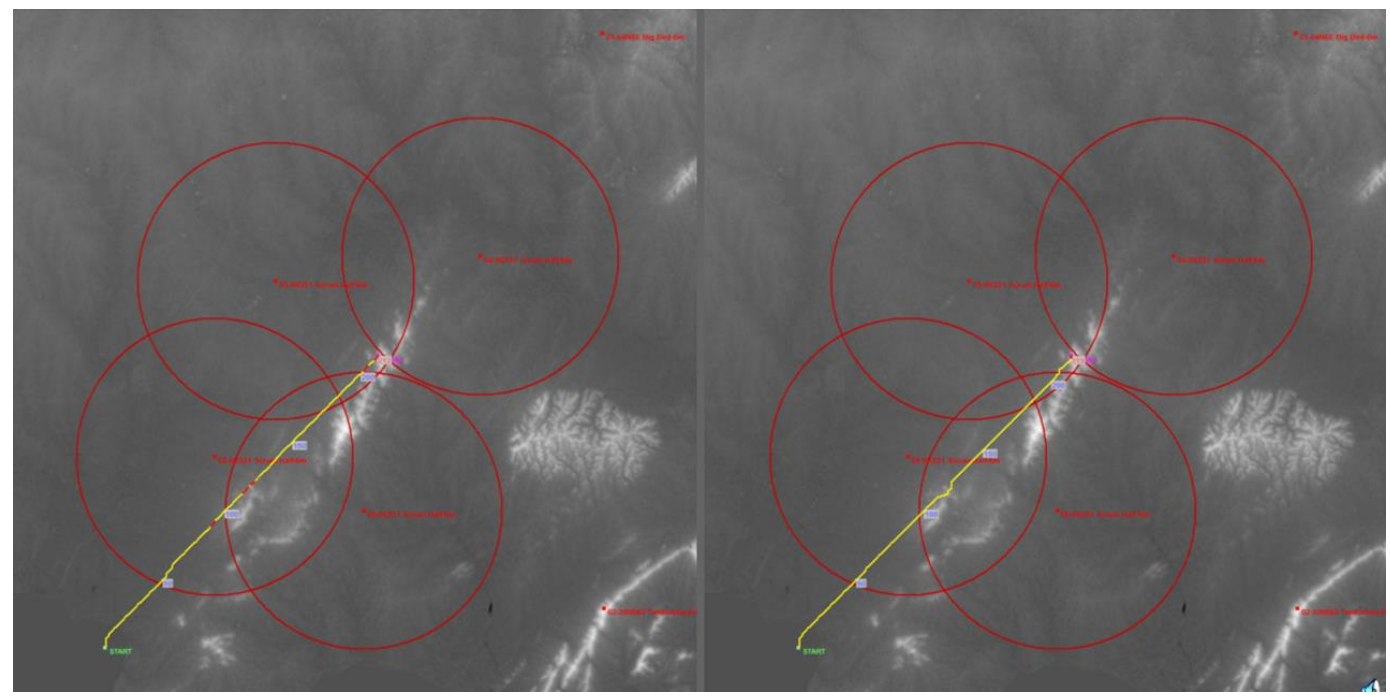

Figure 9. Pathfinding Results for Simulated Mission.

Figure 10 shows another example of a mission on $100 \mathrm{~km}$ wide map defended by both an S-400 battery and Pantsir point defense systems. A $55 \mathrm{~km}$ flight at $30 \mathrm{~m}$ above ground level, or 90 feet above the terrain, takes 3.74 minutes and only the final three seconds are detectable. This is a good result because the missile would not be able to be shot down in those final three seconds according to the Pantsir point defense timing limitations. The main terrain feature the path is able to exploit are the hills to the east of the target airfield, the missile can fly on the far side of the hills from south to north, and only make its final approach and become exposed to the point defense systems in the final three seconds. The missile flies low enough also to be over the curvature of the earth from longer range S-400 observation, despite the fact that the S-400 Big Bird and Tombstone acquisition radars are on elevating platforms which extends their over-the-horizon line of sight range. 


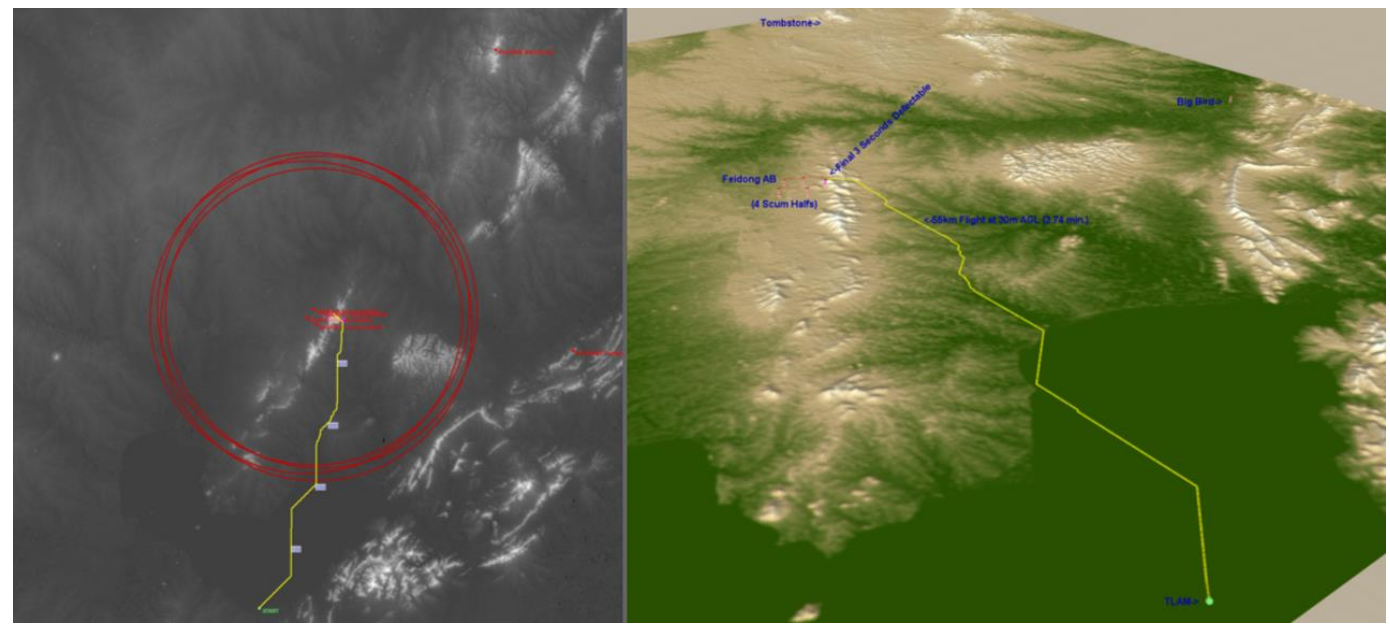

Figure 10. Simulated Airfield Mission Route Results. 


\section{References}

Flight Manual F-105D,F,G, T.O. 1F-105D-1. (1970). United States Air Force

Flight Manual F-111E, T.O. 1F-111E-1. (1973). United States Air Force

Kopp, C., (2009-2011). Engagement and Fire Control Radars (S-band, X-Band, $K u / K / K a$-band). Technical Report APA-TR-2009-0102, Air Power Australia. Retrieved from: http: //www.ausairpower.net/APAEngagement-Fire-Control.html

Kopp, C., (2009-2012). KBP 2K22/2K22M/M1 Tunguska SA-19 Grison /96K6 Pantsir S1 / SA-22 Greyhound SPAAGM. Technical Report APA-TR2009-0703, Air Power Australia. Retrieved from: http://www.ausairpower.net/APA-96K6-Pantsir-2K22-Tunguska.html

Kopp, C., (2009-2012). Kh-55/RKV-500A / 55OK / 55SM/RKV-500B / AS-15 Kent Cruise Missile, in "Soviet/Russian Cruise Missiles. Technical Report APA-TR-2009-0805, Air Power Australia. Retrieved from: http://www.ausairpower.net/APA-Rus-Cruise-Missiles.html

Kopp, C., (2009-2012). Kupol 9K330/9K331/9K332 Tor M/M1/M2 Self Propelled Air Defence System / SA-15 Gauntlet. Technical Report APA-TR-20090705, Air Power Australia. Retrieved from: http://www.ausairpower.net/APA-9K331-Tor.html

Kopp, C., (2005-2012). Tomahawk Cruise Missile Variants; BGM/RGM/AGM109 Tomahawk/TASM/TLAM/GCLM/MRASM. Technical Report APATR-2005-0702, Air Power Australia. Retrieved from: http://www.ausairpower.net/Tomahawk-Subtypes.html

Lynch, D. Jr, Kopp, C., (2008). Multifunctional radar systems for fighter aircraft. Radar Handbook, 3rd Ed, edited by M.I. Skolnik, McGraw Hill Companies, Columbus, OH. pp. 5.1-5.46.

Sullivan, M., (2014). F-35 Joint Strike Fighter: Problems Completing Software Testing May Hinder Delivery of Expected Warfighting Capabilities. Key contributions to this report: Travis Masters, Marvin Bonner, Peter Anderson, Megan Porter, Roxanna Sun and Abby Volk. GAO. GAO-14322. 
Murrow, D.J., (1990). Height Finding and 3D Radar. Radar Handbook, 2nd Ed, edited by M.I. Skolnik. McGraw Hill Companies, Boston, MA. pp. 20.120.40 .

Pelosi M.J., et al, (2012). Range-Limited UAV Trajectory Using Terrain Masking Under Radar Detection Risk. Journal of Applied Artificial Intelligence, Vol.26, Iss.8.

Pettit, R.L., (2004). Low Altitude Threat Evasive Trajectory Generation for Autonomous Aerial Vehicles. M.S. Thesis. Aeronautics and Astronautics Dept., Massachusetts Institute of Technology. Cambridge, MA.

Riedel, F.W. et al, (2010). Guidance and Navigation in the Global Engagement Department. Johns Hopkins APL Technical Digest, Vol. 29, No. 2. pp. 120-125.

Riedel, F.W. et al, (2010). Guidance and Navigation in the Global Engagement Department. Johns Hopkins APL Technical Digest, Vol. 29, No. 2. pp. 129-131

Stimson, G.W., (1998). Introduction to Airborne Radar. 3rd Ed, SciTech Publishing. Washington, DC, 1998, Parts IV - V.

United States Navy Fact File. Tomahawk Cruise Missile. Retrieved from: http://www.navy.mil/navydata/fact_display.asp?cid=2200\&tid=1300\&ct= 2 